-3.5 to $11.7 ; 26.8$; CI, 17.6 to 36.8 , respectively), a subjective visual analogue scale for alertness $(-1.3$; CI, -3.7 to $1.2 ;-2.5$; CI, -4.8 to $-0.2 ;-5.3 ; \mathrm{CI},-7.7$ to -2.9 , respectively) and saccadic peak velocity (-14.3; CI, 33.5 to $4.8 ;-10.9$; CI, -29.0 to $7.1 ;-25.4$ (CI, -44.2 to -6.6 , respectively), a marker for sedation. Mecamylamine was well tolerated and had linear pharmacokinetics over the dose range tested. Conclusion: The pharmacological challenge model with mecamylamine $20 \mathrm{mg}$ in healthy subjects leads to a reproducible pattern of cognitive disturbance that is nicotinic receptor specific. This model may be more suitable for proof of pharmacology and dose finding studies of nicotinic receptor agonists than the frequently used scopolamine model.

\section{BENEFIT-RISK OF VKA FOR ATRIAL FIBRILLATION BEFORE DOAC: A COHORT STUDY IN A CLAIMS AND HOSPITALIZATION DATABASE}

P. Blin $^{1,2}$; C. Dureau-Pournin ${ }^{1,2}$; R. Lassalle ${ }^{1,2}$; A. Abouelfath ${ }^{1,2}$; C. Droz-Perroteau ${ }^{1,2}$; and N. Moore ${ }^{1,3}$

${ }^{1}$ INSERM CIC1401, Bordeaux, France; ${ }^{2}$ ADERA, Pessac, France; and ${ }^{3}$ University of Bordeaux, Bordeaux, France

Background: Vitamin K antagonists (VKA) are a major iatrogenic cause of haemorrhage and hospital admissions, but the incidence of bleeding during VKA exposure for atrial fibrillation (AF), as well as ischemic event, is not well established in real life and before introduction of direct oral anticoagulants (DOAC) for non-valvular AF (NVAF).

Material and Methods: Cohort study of new VKA users between 2007 and 2011, with 2-year history and 3-year follow-up censored at end of 2012, was designed in the EGB, a 1/97 random sample of the French national health care claims and hospitalization database. The AF population was defined as patients with full coverage for $\mathrm{AF}$, hospitalization, or probabilistic AF information in the database and the NVAF population as patients with AF diagnosis information (full coverage, hospitalization), without valvular disease history or other probable cause of VKA prescription. Outcomes were the first hospitalization for bleeding, arterial thrombotic event (ATE), acute coronary syndrome (ACS), and death. Incidence rate of outcomes was estimated during VKA exposure.

Results: Among 8894 patients identified, 3530 were classified in AF population. Half were male ( $51 \%$ ) with a mean age of 75 years, $87 \%$ had a $\mathrm{CHA}_{2} \mathrm{DS}_{2}$-VASc score $\geq 2$, and $12 \%$ a HAS-BLED score $>3$. The incidence rate of bleeding was 29 patients (95\% CI, 24-34) for 1000 PY exposed to VKA, including 6 (95\% CI, 4-8) cerebral, 10 (95\% CI, 7-13) digestive, and 14 (95\% CI, 10-17) other bleeds. Incidence rates were 16 (95\% CI, 12-19) for ACS, 14 (95\% CI, 11-18) for ATE, and 39 (95\% CI, 33-45) deaths. Patient characteristics and incidence rates were very close for the 1813 patients with NVAF population criteria. For this last population, incidence rates were 29 (95\% CI, 23-34) for bleeding, 15 (95\% CI, 11-19) for ACS, 14 (95\% CI, 10-18) for ATE, and 37 (95\% CI, 31-43) deaths. Conclusions: This study provides background reference for bleeding, ischemic events, and deaths before introduction of DOAC for NVAF with quite same frequency for AF and NVAF populations.

\section{IMPACT OF ADVANCED AGE ON THE PHARMACOKINETICS AND PHARMACODYNAMICS OF ROCURONIUM IN PATIENTS UNDERGOING ELECTIVE SURGERY}

N.V. de Moraes $^{1}$; V.L. Lanchote ${ }^{2}$; G.O. Filgueira ${ }^{2}$; B.C.P. Lopes ${ }^{2}$; J.S. Lepera ${ }^{1}$; and G.R. Lauretti ${ }^{2,3}$

${ }^{1}$ Faculdade de Ciências Farmacêuticas, Universidade Estadual Paulista, Araraquara, Brazil; ${ }^{2}$ Faculdade de Ciências
Farmacêuticas de Ribeirão Preto, Universidade de São Paulo, Ribeirão Preto, Brazil; and ${ }^{3}$ Faculdade de Medicina de Ribeirão Preto, Universidade de São Paulo, Ribeirão Preto, Brazil

Introduction: Increasing age are associated with greater morbidity and mortality after anesthesia. Rocuronium (ROC), a neuromuscular blocking agent used in surgical procedures, is primarily eliminated by biliary and renal excretion. The objective of this study is to evaluate the impact of advanced age on ROC pharmacokinetics (PK) and pharmacodynamics (PD) in ASA I-III patients undergoing elective surgeries.

Material and Methods: Adult patients (aged 20-50 years, $\mathrm{n}=13$ ) and elderly patients (aged $65-85$ years, $n=13$ ) submitted to surgery under general anesthesia were investigated. All patients were induced with individual intravenous doses of midazolam, rocuronium, fentanyl and propofol. Serial blood samples were collected up to 360 minutes after administration of ROC. Rocuronium-induced neuromuscular block was monitored by train of four stimulation of the adductor muscle of the thumb on the ulnar nerve at the same times of blood collection. The plasma concentration of ROC was analyzed by liquid chromatography coupled to mass spectrometry with electrospray ionization using positive ion mode. The pharmacokinetic parameters area under the curve normalized by dose (AUC/ dose) and clearance were calculated by non-compartmental analysis. The relationship between rocuronium plasma concentration and the neuromuscular blockade was described by a sigmoid maximum effect model for each patient. Data are presented as median (interquartile range).

Results: Elderly patients presented decreased clearance $(2.1 \mathrm{~mL}$. $\mathrm{kg}^{-1} \mathrm{~min}^{-1}[1.6-2.5]$ vs $\left.2.6 \mathrm{~mL} . \mathrm{kg}^{-1} \mathrm{~min}^{-1}[2.2-3.2]\right)$ and increased AUC/dose (476.1 $\mu \mathrm{g} \cdot \mathrm{min} \cdot \mathrm{mL}^{-1} /\left[\mathrm{mg} \cdot \mathrm{kg}^{-1}\right]$ ([396.4-621.9] vs $386.5 \mu \mathrm{g}$. min. $\left.\mathrm{mL}^{-1} /\left[\mathrm{mg}_{\mathrm{kg}}{ }^{-1}\right][308.5-459.9]\right)$ compared to adults. The concentrations required to achieve $50 \%$ of maximum neuromuscular block (EC50) were similar for adult $(502.5 \mathrm{ng} / \mathrm{mL})$ and elderly $(587.7 \mathrm{ng} / \mathrm{mL})$ patients.

Conclusion: Elderly patients showed increased AUC/dose and reduced total clearance compared to adult patients, probably due to the age-related reduced creatinine clearance. Differences in the PK-PD properties of ROC in elderly population are due to changes in drug disposition rather than to alterations in the sensitivity to the drug.

\section{CLUSTERING OF RARE MEDICAL CONDITIONS BASED ON CLINICAL FEATURES WHICH DETERMINE APPLICABILITY OF INVESTIGATIVE DESIGNS AND METHODS TO THEIR STUDY}

C. Pontes ${ }^{1}$; J.M. Fontanet ${ }^{1}$; M. Gomez-Valent ${ }^{1}$; J. Rios Guillermo ${ }^{1}$; R. Vives Vilagut ${ }^{1}$; R. Morros ${ }^{1}$; J. Martinalbo ${ }^{2}$; J. Torrent-Farnell ${ }^{1}$; and F. Torres

${ }^{1}$ Universitat Autònoma de Barcelona, Barcelona, Spain; and ${ }^{2}$ European Medicines Agency, London, United Kingdom

Background: The EU legislation determines that market access to new drugs requires the same level of evidence regardless of whether they are intended for rare or highly prevalent diseases, but the reality is that regulators often have to take decisions on orphan medicinal products (OMP) based on limited amounts of evidence. Clinical development for new orphan drugs with a cost-efficient design and analysis, an optimal fit to needs of patients, and reliable and efficient to decision making is thus of utmost importance to patients, regulators, and the pharmaceutical industry. Within an international FP7 collaboration (ASTERIX project), we have established a systematic and clinically based clustering in order to propose designs and analyses for trials conducted in small populations. 